【研究简报】

\title{
用耦合的电子振动光谱探测稀土离子 的配位环境
}

\author{
李润华 孟建新 周建英 曾学然罗 琦 周达君
}

(中山大学超块速澈光光诸学国家重点实输空,广州 510275)

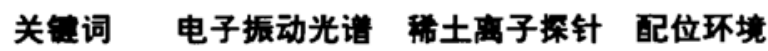

随着世界人口的急剧增长, 粮食已经成为一个至关重要的问题. 曾有报道表明, 稀土元素 能够增加农作物的产量和刺激动植物的生长 ${ }^{[1]}$. 但是, 过量的稀土元素是否对人体有害, 是 一个直接影响到我国未来稀土农业决策的问题. 对这一问题的深入认识依赖于对稀土元素在 生物体中配位环境的详细了解. 然而 X-射线的结构分析方法, 却难以应用于生物样品, 因为 生物样品通常不具备单一的晶体结构; 核磁共振的方法又要求样品有较高的浓度. 与这些方 法相比, 光谱学的方法具有良好的选择性和较高的检测灵敏度, 而且不受样品形态的限制, 因 此有必要发展光谱学的技术来探测生物样品中稀土元素的配位环境.

在稀土离子的配合物中, 可以观测到稀土离子的电子跃迁与环境配体振动耦合而成的一 种跃迁, 即耦合的电子振动跃迁 (cooperative vibronic transition, 以下简称 CV 跃迁) ${ }^{[2]}$. CV 跃 迁与其对应的电子跃迁的频率差等于配体的红外振动频率, 因此通过比较这一频率差与红外 光谱的数据可以确定在稀土离子第一配位层的配体种类. 我们正是利用这一技术探测到了一 些生物样品中 $\mathrm{PO}_{4}^{3-}, \mathrm{COO}^{-}$的配位 ${ }^{[3,4]}$.

然而要了解稀土离子的配位环境, 仅仅知道配体的种类是不够的, 还必须知道在稀土离子 周围的每一种配体的数目. Horrocks 等人发展的用苂光寿命测量配体数目的方法由于依赖于 水分子大的退激活系数, 因此只能用于测量水分子的配位数; 而且为了确定无水分子退激活时 的苂光寿命, 还必须用重水来替代水, 因而增加了这一技术的复杂性 ${ }^{[5,6]}$.

本文将介绍一种新的利用 CV 光谱测量配体数目的原理与技术, 这一技术简单易行, 所有 能够探测到 $\mathrm{CV}$ 光谱的配体的数目都能够用这一技术来确定.

\section{1 理论}

Stavola 等人以双中心模型并利用一阶微扰处理发展了 CV 跃迁理论, 但他们仅仅考虑了 一个配体 ${ }^{[2]}$. 如果同一种类的配体的数目不止一个, 则可以利用由 Faulkner 和 Richardson 所 提出的独立系统模型 (模型的正确性已由他们的理论与实验结果的一致性得到了验证) ${ }^{[7]}$. 在这一模型中, 每一个配体被看成只与中心离子通过库仑力相互作用, 配体之间没有相互作 用, 这样, $n$ 个相同配体的 CV跃迁的振子强度可以线性迭加. 因此, 如果仅仅考虑电偶极相 互作用,则由 $n$ 个相同配体参与的 CV 跃迁的振子强度可以表示成为 ${ }^{[2]}$

$$
P_{\mathrm{CV}}^{(n)} \propto n \frac{1}{2 J+1} \frac{1}{R^{6}} \Xi^{2}(1,2)\left|\left\langle J\left\|\mathrm{U}^{(2)}\right\| J^{\prime}\right\rangle\right|^{2}\left|\left\langle 0 \mu\left|\mathrm{M}^{(1)}(B)\right| 0 \mu^{\prime}\right\rangle\right|^{2},
$$

这里, $\Xi(1,2)$ 是常数; $R$ 是稀土离子与配体电荷中心的距离; $J, J^{\prime}$ 是电子能级的角量子数; 
$0 \mu, 0 \mu^{\prime}$ 是配体振动态的量子数; $\mathrm{U}^{(2)}$ 是一个二阶单位操作算符; $\mathrm{M}$ 是张量操作算符, 其定义 见文献 [2]中 (3.8)式, 它的矩阵元的平方表征了配体 B 的红外跃迁振子强度.

根据熟知的由 Judd 和 Ofelt 发展的 “强迫电偶极跃迁理论” ${ }^{[8,9]}$, 稀土离子的电偶极跃迁 的振子强度为 ${ }^{[8]}$

$$
P_{\text {ele }} \propto \frac{1}{2 J+I} \Xi^{(2)}(1,2)\left|\left\langle J\left\|\mathrm{U}^{(2)}\right\| J^{\prime}\right\rangle\right|^{2},
$$

所以,

$$
P_{\mathrm{CV}}^{(n)} / P_{\text {ele }} \propto \frac{n}{R^{6}}\left|\left\langle 0 \mu\left|\mathrm{M}^{(1)}(B)\right| 0 \mu^{\prime}\right\rangle\right|^{2} .
$$

对于不同配合物中相同的配体, $\left\langle 0 \mu\left|\mathrm{M}^{(1)}(B)\right| 0 \mu^{\prime}\right\rangle$ 可近似作为一个常数, 因而从 CV 跃迁与 稀土离子电子跃迁的强度之比可以得到配体数目的信息.

\section{2 实验和结果}

采用文献 $[10]$ 描述的实验系统并利用 时间分辨的处理技术, 研究了 $\mathrm{Tb}^{3+}$ 的五种 水合盐中 $\mathrm{Tb}^{3+}$ 与水分子的 $\mathrm{CV}$ 跃迁谱(激 发谱, 下同), 其峰值强度 (以 $I_{\mathrm{CV}}$ 表示) 与 $\mathrm{Tb}^{3+}$ 的 ${ }^{7} \mathrm{~F}_{6} \rightarrow{ }^{5} \mathrm{D}_{4}$ 跃迁的电子谱的峰值强 度 (以 $I_{\text {ele }}$ 表示) 之比列于表 1 中. 假定 $\mathrm{TbCl}_{3} \cdot 6 \mathrm{H}_{2} \mathrm{O}$ 中 6 个配位水为已知, 可以计

表 1 五种水合盐的实验数据和测得的水配位数

\begin{tabular}{lcll}
\hline \multicolumn{1}{c}{ 样品 } & $\left.N^{s}\right)$ & \multicolumn{1}{c}{$I_{\mathrm{cv}} / I_{\mathrm{de}}$} & $N_{\mathrm{cv}}{ }^{\mathrm{b}}$ \\
\hline $\mathrm{TbCl}_{3} \cdot 6 \mathrm{H}_{2} \mathrm{O}$ & 6 & 0.0067 & 6 \\
$\mathrm{~Tb}_{2}\left(\mathrm{SO}_{4}\right)_{3} \cdot 8 \mathrm{H}_{2} \mathrm{O}$ & 4 & 0.0042 & 3.8 \\
$\mathrm{~Tb}\left(\mathrm{NO}_{3}\right)_{3} \cdot 5 \mathrm{H}_{2} \mathrm{O}$ & 3 & 0.00355 & 3.2 \\
$\mathrm{~Tb}_{2}\left(\mathrm{C}_{2} \mathrm{O}_{4}\right)_{3} \cdot 10 \mathrm{H}_{2} \mathrm{O}$ & 3 & 0.0037 & 3.3 \\
$\mathrm{~Tb}\left(\mathrm{C}_{6} \mathrm{H}_{4} \mathrm{O}_{2} \mathrm{~N}\right)_{3} \cdot 2 \mathrm{H}_{2} \mathrm{O}$ & 2 & 0.00234 & 2.1 \\
\hline
\end{tabular}

a) 用 X-射线测得的水配位数; b) 用 CV 谱测得的水配位数 品的水配位数. 表 1 还列出了 5 种水合算出其他样盐 $\mathrm{X}$-射线分析的水配位数, 可以看出用 $\mathrm{CV}$ 谱测得的结果近似与用 $\mathrm{X}$-射线分析的结果一致. 我们还研究了 6 种含不同比例水和重水 的 $0.1 \mathrm{MTbCl}_{3}$ 溶液中钱离子与水 (重水)的 $\mathrm{CV}$ 跃迁谱, 实验数据列于表 2 . 同样, 假定 $\mathrm{TbCl}_{3}$ 的纯水 (重水) 溶液中 9 个配位水 (重水) 为已知, 可以计算出其他样品中水 (重水)的配位数.

表 2 用 $\mathrm{CV}$ 谱测得的六种含不同比例水和重水的 $0.1 \mathrm{MTbCl}_{3}$ 溶液中水(重水)的配位数

\begin{tabular}{cclcc}
\hline 样品中水的含量/\% & $I_{\mathrm{Cv}}\left(\mathrm{H}_{2} \mathrm{O}\right) / I_{\mathrm{db}}$ & $N_{\mathrm{H}_{2} \mathrm{O}}$ & $I_{\mathrm{cv}}\left(\mathrm{D}_{2} \mathrm{O}\right) / I_{\text {de }}$ & $N_{\mathrm{D}_{2} \mathrm{O}}$ \\
\hline 100 & 0.01 & 9 & 0 & 0 \\
80 & 0.00822 & 7.4 & 0.0031 & 1.8 \\
60 & 0.00567 & 5.1 & 0.0066 & 3.8 \\
40 & 0.00398 & 3.6 & 0.009 & 5.2 \\
20 & 0.00189 & 1.7 & 0.0126 & 7.3 \\
0 & 0 & 0 & 0.0156 & 9 \\
\hline
\end{tabular}

我们还用苂光寿命方法测量了以上溶液中水分子的配位数,并间接地得出了重水的配位 数, 结果列于表 3. 比较两种技术的结果可以看出在实验误差的范围内基本一致.

表 3 用苂光寿命方法测得的 6 种溶液中水和重水的配位数

\begin{tabular}{ccccc}
\hline 样品中水的含量/\% & $\tau_{\text {obad }} / \mathrm{ms}$ & $k_{\text {doded }} / \mathrm{ms}^{-1}$ & $N_{\mathrm{D}_{2} \mathrm{O}}$ & $N_{\mathrm{D}_{2} \mathrm{O}}=9-N_{\mathrm{H}_{2} \mathrm{O}}$ \\
\hline 100 & 0.403 & 2.48 & 9 & 0 \\
80 & 0.483 & 2.07 & 7.3 & 1.7 \\
60 & 0.637 & 1.57 & 5.2 & 3.8 \\
40 & 0.826 & 1.21 & 3.7 & 5.3 \\
20 & 1.282 & 0.78 & 1.9 & 7.1 \\
0 & 3.125 & 0.32 & 0 & 0 \\
\hline
\end{tabular}


另外我们还配制了两种不同浓度的由 $\mathrm{TbCl}_{3}$ 和 $\mathrm{NaCH}_{3} \mathrm{COO}$ 组成的水溶液,在这两种溶液 中, $\mathrm{Tb}^{3+}$ 与 $\mathrm{COO}^{-}$离子的摩尔浓度比分别为 $1: 1$ 和 $1: 2$. 实验记录了 $\mathrm{COO}^{-}$和水与 $\mathrm{Tb}^{3+}$ 离子 的 $\mathrm{CV}$ 跃迁谱,数值结果和测得的配位的水分子以及 $\mathrm{COO}^{-}$离子数列于表 4 中.

表 4 用 $\mathrm{CV}$ 谱消得的两种 $\mathrm{Tb}\left(\mathrm{CH}_{3} \mathrm{COO}\right)_{3}$ 溶液中配位的水分子和 $\mathrm{COO}^{-}$离子数

\begin{tabular}{ccccc}
\hline $\mathrm{Tb}^{3+}: \mathrm{COO}^{-}$ & $I_{\mathrm{cv}}\left(\mathrm{H}_{2} \mathrm{O}\right) / I_{\text {cte }}$ & $I_{\mathrm{cv}}\left(\mathrm{COO}^{-}\right) / I_{\mathrm{ct}}$ & $N_{\mathrm{H}_{2} \mathrm{O}}$ & $N_{\mathrm{CoO}^{-}}$ \\
\hline $1: 1$ & 0.0071 & 0.0712 & 6.4 & 1.0 \\
$1: 2$ & 0.00567 & 0.119 & 5.1 & 1.65 \\
\hline
\end{tabular}

\section{3 讨论}

在表 1 中, 固体样品的测量误差主要来自 $R$ 的影响, 从结果可以看出, 在这几个样品中 $R$ 相差并不大. 由于固体样品中配位数应为整数, 所以如果将绝对误差 0.5 作为误差上限, 采取 四舍五入,则用 $\mathrm{CV}$ 谱测得的这几个固体样品的水配位数与 X-射线分析的结果完全一致.

在稀溶液中, 由于存在快速的配体交换, $R$ 应为一平均值, 而且在不同的样品中该值应近 似不变, 其影响可以忽略. 因此我们可以定义一个常数 $C$, 其物理意义为单个配体导致的 $\mathrm{CV}$ 跃迁与电子跃迁的峰值强度比. 这样, 对于某一配体, 一旦 $\mathrm{C}$ 确定, 其配位数 $n$ 就可以由下式 简单地确定(表 4 中的水配位数就是这样得到的):

$$
n=\frac{I_{\mathrm{cV}} / I_{\mathrm{ele}}}{\mathrm{C}} \text {. }
$$

由表 2 和表 3 可以得出: $C_{\mathrm{H}_{2} \mathrm{O}}=0.00111 ; C_{\mathrm{D}_{2} \mathrm{O}}=0.00173 ; C_{\mathrm{COO}^{-}}=0.0716$.

表 4 中配位的 $\mathrm{COO}^{-}$离子数是这样确定的: 由于 $\mathrm{Tb}^{3+}$ 离子在水溶液中的配位数一般为 $8 \sim 9$, 在 $1: 1$ 的样品中, 已经得出水的配位数为 6.4 , 而这一样品中一个 $\mathrm{Tb}^{3+}$ 离子最多只能与 一个 $\mathrm{COO}^{-}$离子配位; 又由于一个 $\mathrm{COO}^{-}$离子能够提供两个配位数, 所以得到在 $1: 1$ 的样品 中, 参与配位的 $\mathrm{COO}^{-}$离子数为 1 . 而在 $1: 2$ 的样品中, 总配位数仍应为 8.4 , 以此减去水配位 数再除以 2 即是参与配位的 $\mathrm{COO}^{-}$离子的数目. 可以看出, 两种溶液中, $I_{\mathrm{CV}}\left(\mathrm{COO}^{-}\right) / I_{\mathrm{ele}}$ 值之 比 $(0.119 / 0.0712=1.67)$ 近似等于用这种方法得到的配位的 $\mathrm{COO}^{-}$离子数之比.

最后有必要提一下, Blasse 等人误解了 Judd 得到的关于 $\mathrm{XCl}_{6}^{-}$体系电子振动跃迁的理论 结果 ${ }^{[11,12]}$, 文献 [11] 的方程 (12) 中因子 $\left(g+6 \alpha R^{-3}\right)$ 中的 6 是 $2 \times Z_{\mathrm{RE}}$ (这里, $Z_{\mathrm{RE}}=3$, 是稀土 离子的净电荷), 而不是配体的数目. 因此电子振动跃迁的振子强度不是跟 $\left(g+n a R^{-3}\right)^{2}$ 成 正比, 而是跟 $n$ 成正比, 这里, $n$ 是配体数目.

\section{4 结论}

用稀土离子的 $\mathrm{CV}$ 光谱可以确定配体的种类和数目. 这一技术不仅提供了一种简单的、 无需重水替代的测量水配位数目的方法, 而且还大大扩展了可以直接测量配位数目的配体的 种类. 这一光谱学技术不受样品形态的限制, 探测灵敏度高. 它除了能够作为一般样品的结 构分析方法之外, 更重要的是能够作为生物样品中金属离子配位环境的光谱探针. 因此这一 技术对研究稀土元素的生命功能具有特殊的意义. 